\title{
LSST Camera Optics Design
}

V. J. Riot, S. Olivier, B. Bauman, S. Pratuch, L. Seppala, D. Gilmore, J. Ku, M. Nordby, M. Foss, P. Antilogus, N. Morgado

May 29, 2012

SPIE Astronomy 2012 - Space, Ground-based, and Airborne Telescopes, Observatory Operations, Adaptive Optics Amsterdam, Netherlands July 1, 2012 through July 6, 2012 
This document was prepared as an account of work sponsored by an agency of the United States government. Neither the United States government nor Lawrence Livermore National Security, LLC, nor any of their employees makes any warranty, expressed or implied, or assumes any legal liability or responsibility for the accuracy, completeness, or usefulness of any information, apparatus, product, or process disclosed, or represents that its use would not infringe privately owned rights. Reference herein to any specific commercial product, process, or service by trade name, trademark, manufacturer, or otherwise does not necessarily constitute or imply its endorsement, recommendation, or favoring by the United States government or Lawrence Livermore National Security, LLC. The views and opinions of authors expressed herein do not necessarily state or reflect those of the United States government or Lawrence Livermore National Security, LLC, and shall not be used for advertising or product endorsement purposes. 


\title{
LSST Camera Optics Design
}

Scot S. Olivier ${ }^{\mathrm{a}}$, Vincent J. Riot*a, David K. Gilmore ${ }^{\mathrm{b}}$, Brian Bauman ${ }^{\mathrm{a}}$, Steve Pratuch ${ }^{\mathrm{a}}$, Lynn Seppala ${ }^{\mathrm{a}}$, John $\mathrm{Ku}^{\mathrm{b}}$, Martin Nordby ${ }^{\mathrm{b}}$, Mike Foss ${ }^{\mathrm{b}}$, Pierre Antilogus ${ }^{\mathrm{c}}$, Nazario Morgado ${ }^{\mathrm{d}}$, Benoit Sassolas ${ }^{\mathrm{d}}$, Raffaele Flaminio ${ }^{\mathrm{d}}$, Christophe Michel $^{\mathrm{d}}$

${ }^{a}$ Lawrence Livermore National Laboratory, 7000 East Ave., Livermore, CA, USA 94550

${ }^{b}$ SLAC National Accelerator Laboratory, 2575 Sand Hill Rd., Menlo Park, CA USA 94025

'Institut National de Physique Nucléaire et de Physique des Particules (IN2P3), 3 rue Michel-Ange, 75794 Paris Cedex 16, France

${ }^{\mathrm{d}}$ Laboratoire des Matériaux Avancés (LMA), IN2P3/CNRS, F-69622 Villeurbanne, Lyon, France

\begin{abstract}
The Large Synoptic Survey Telescope (LSST) uses a novel, three-mirror, telescope design feeding a camera system that includes a set of broad-band filters and three refractive corrector lenses to produce a flat field at the focal plane with a wide field of view. Optical design of the camera lenses and filters is integrated in with the optical design of telescope mirrors to optimize performance. We discuss the rationale for the LSST camera optics design, describe the methodology for fabricating, coating, mounting and testing the lenses and filters, and present the results of detailed analyses demonstrating that the camera optics will meet their performance goals.
\end{abstract}

Keywords: LSST, telescope, filters, optical mounts

\section{INTRODUCTION}

The LSST camera refractive optics system consists of three fused silica lenses (L1, L2, L3) with diameters of $1.6 \mathrm{~m}, 1.1$ $\mathrm{m}$ and $0.73 \mathrm{~m}$ that correct for field aberrations, along with interchangeable filters with diameters of $0.78 \mathrm{~m}$ that give spectral coverage from the UV to near IR (See Figure 1). The optics design meets all design requirements using fused silica substrates. The Corning manufacturing process for fused silica produces glass of the required size and quality. Although the required lenses are large, a substantial industrial base exists for fabricating them, and the LSST requirements are consistent with optical elements that have been produced for other programs. A key aspect of the fabrication involves the use of relatively simple null tests that have been factored into the optical design. All null tests use a retro-reflecting mirror to test the lenses in double pass transmission. A perfect point source is re-imaged onto itself with a wavefront error less than $1 / 20$ wave at $633 \mathrm{~nm}$

The LSST camera optics along with $0.64 \mathrm{~m}$ diameter detector array are housed in a canister, $\sim 1.6 \mathrm{~m}$ in diameter, the size of the largest lens. The filter coating is a relatively sophisticated multi-layer interference coating that is designed to transmit only light with wavelengths in a specified band, and to reject light at other wavelengths with a specified fidelity. The main challenge is to deposit uniform coatings with the desired characteristics on the large, curved substrates typically with about 100 layers on each surface. The baseline design has the first surface of the filters concentric about the chief ray in order to keep the angles of the light rays passing through the filters as uniform as possible over the entire range of field positions.

The optical elements are held in a static position by three primary structures. The first structure is a lightweight toroidal ring that holds L1 and L2, and maintains the correct distance between them to maintain the optical quality of the input beam. This ring is attached to the camera body through six low CTE adjustable struts. The second structure is the L3 assembly, held in place by a thermally isolated ring attached to the cryostat housing. L3 is the vacuum barrier to the cryostat so more structural and stress analysis has been performed on this lens. The central thickness is specified in order to provide a significant safety margin for potential fracture of L3 due to the pressure differential. Finally, an automated filter changing mechanism with a repeatable interface that will hold any of the five in-camera filters in the correct aligned optical position above the focal plane.

*riot1@llnl.gov; phone 1925 422-9798; 1lnl.gov 


\section{REQUIREMENTS}

\subsection{Optics prescription}

The current baseline optical design for the LSST is a modified Paul-Baker three-mirror telescope [1][2] that includes an 8.4-meter primary mirror (M1) which is cast with a 5.0-m tertiary mirror (M3); the casting together of primary/tertiary was also seen in Rumsey [3]. After a first reflection on M1, the optical beam converges on the 3.4-m convex secondary mirror (M2). From M2, the reflected beam diverges slightly toward M3, and is then focused toward the camera located in front of M2 on the optical axis (see Figure 1). The current design employs three aspheric mirrors. The three-mirror telescope system delivers, without the camera corrector optics, an on axis spherical wavefront that will greatly help in initial assembly and alignment. Specification of the telescope parameters is given in Table 1.

Table 1. LSST Specifications.

\begin{tabular}{|l|l|}
\hline Design type & Modified Paul-Baker \\
\hline Entrance pupil & 8.4 meters with 5.0 meter $(\sim 62 \%)$ central obscuration \\
\hline F-number & $\mathrm{f} / 1.2345$ \\
\hline Field of view & $3.5^{\circ}$ diameter, with additional corner rafts for wavefront sensing \\
\hline Approximate FHWM image blur & 0.2 arcsec FWHM \\
\hline Detector size & $640 \mathrm{~mm}$ diameter \\
\hline Pixel size & $10 \mu \mathrm{m}=0.2$ arcsec \\
\hline Wavelength range & 6 bands within $330-1060 \mathrm{~nm}$ \\
\hline
\end{tabular}

The LSST makes use of a three-lens corrector [4] to correct for chromatic aberrations imparted by the sensor vacuum window as well as improve image quality on a flat field. The baseline LSST Camera optics design consists of three refractive lenses with clear aperture diameters of $1.550 \mathrm{~m}(\mathrm{~L} 1), 1.100 \mathrm{~m}$ (L2) and $0.722 \mathrm{~m}$ (L3) and six interchangeable (five in camera), broad-band filters with clear aperture diameters of $0.75 \mathrm{~m}$, which provide spectral coverage from the UV to the near IR. L2 and L3 each have one aspheric surface. See Figure 1 and Table 1 for a description of the optics.

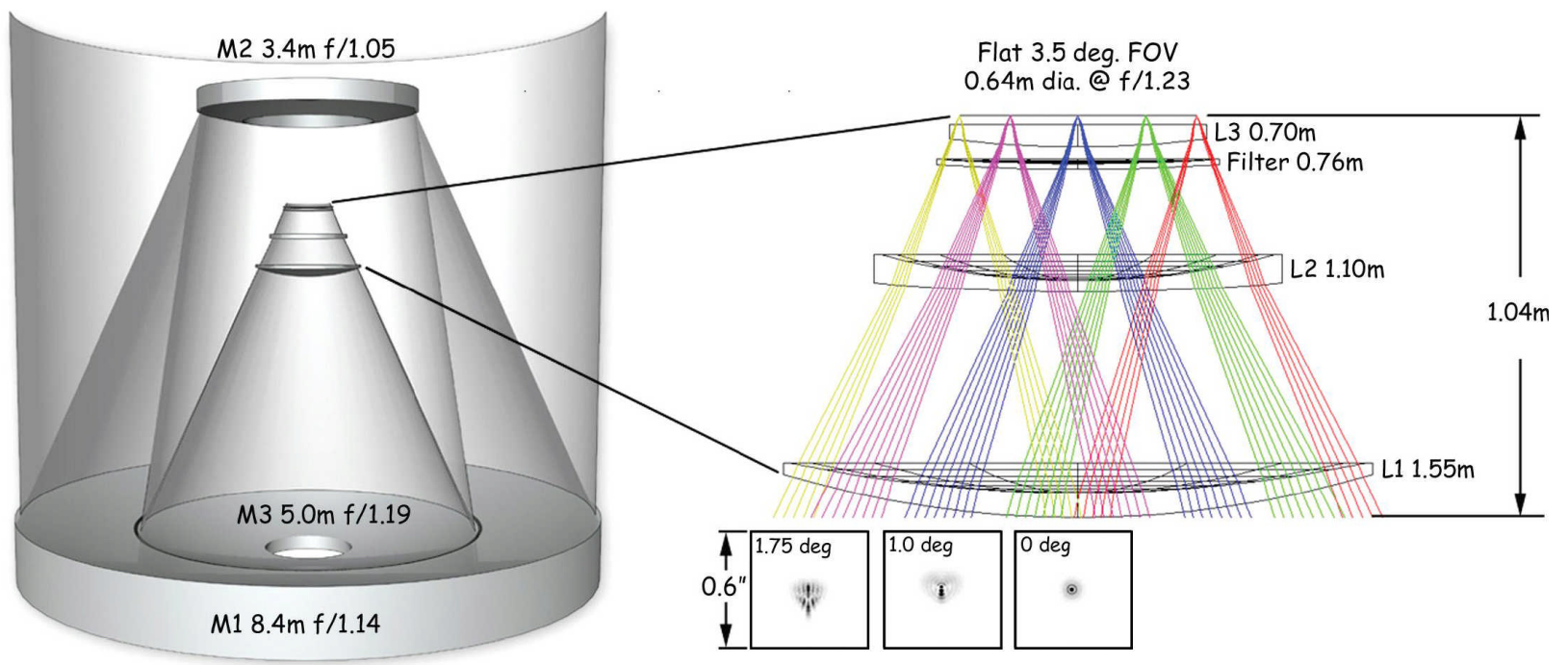

Figure 1. LSST optical design includes 3 large mirrors, 3 large lenses and a set of 6 large transmission filters.

The smallest lens, L3, with a clear aperture of $0.722 \mathrm{~m}$, is also the vacuum barrier for the cryostat containing the detector array. There is a $2.85 \mathrm{~cm}$ gap between the second surface of L3 and the focal plane. The L3 central thickness (see table 2 ) is specified in order to provide adequate safety margin for potential fracture of L3 due to the pressure differential. Empirical data shows that a thickness ratio of $\sim 12$ is adequate to provide this safety margin, which yields a thickness of $\sim 6 \mathrm{~cm}$ for this lens. The filters are designed to be fabricated using multi-layer dielectric interference coatings deposited on fused silica substrates. The baseline design has the first surface of the filters concentric about the pupil (i.e., all chief rays are normal to the surface) in order to keep the angles of the light rays passing through the filters as uniform as possible over the entire range of field positions. The central thickness and the curvature of the second surface are 
optimized for image quality. The minimum center thickness is $1.35 \mathrm{~cm}$ for the y-band filter, and the maximum is 2.62 $\mathrm{cm}$ for the u-band filter. Detailed parameters for the camera optics are given in Table 2.

Table 2. LSST camera optical element design parameters.

\begin{tabular}{|l|r|r|r|r|r|r|r|r|r|}
\cline { 2 - 11 } \multicolumn{1}{c|}{} & \multicolumn{3}{c|}{ Lenses } & \multicolumn{1}{c|}{ Filters } \\
\hline Clear Aperture Dims & \multicolumn{1}{c|}{ L1 } & \multicolumn{1}{c|}{ L2 } & \multicolumn{1}{c|}{ L3 } & \multicolumn{1}{c|}{ U } & \multicolumn{1}{c|}{ G } & \multicolumn{1}{c|}{ R } & I & \multicolumn{1}{c|}{ Z } & y \\
\hline Surface 1 vertex to FPA & 1031.950 & 537.080 & 88.500 & 157.500 & 157.500 & 157.500 & 157.500 & 157.500 & 157.500 \\
\hline Surface 2 vertex to FPA & 949.720 & 507.080 & 28.500 & 130.900 & 136.000 & 139.600 & 141.800 & 143.100 & 143.900 \\
\hline Center thickness & 82.230 & 30.000 & 60.000 & 26.600 & 21.500 & 17.900 & 15.700 & 14.400 & 13.600 \\
\hline Clear aperture rad. & 775.000 & 551.000 & 361.000 & 378.000 & 378.000 & 378.000 & 378.000 & 378.000 & 378.000 \\
\hline Surface 1 spherical rad. & 2824.000 & Flat & 3169.000 & 5632.000 & 5632.000 & 5632.000 & 5632.000 & 5632.000 & 5632.000 \\
\hline Surface 2 spherical rad. & 5021.000 & 2529.000 & 13360.000 & 5530.000 & 5576.000 & 5606.000 & 5623.000 & 5632.000 & 5640.000 \\
\hline Edge Thickness & 28.974 & 88.297 & 30.152 & 26.856 & 21.640 & 17.964 & 15.722 & 14.400 & 13.580 \\
\hline
\end{tabular}

*All dimensions in mm except as noted

"Approx Physical Dims" for reference only

Five filters are resident in the Camera; any two filters can be changed in less than 90 seconds. One of the six filters will be stored off-camera at any given time and can be exchanged with any of the five on-camera filters during a daytime servicing operation.

\subsection{Optics mechanical requirements}

The optical elements experience structural loads from four potential sources: inertial accelerations, thermal distortions, pressure differentials, and internal stresses during manufacturing. Each of these sources will degrade the image quality delivered to the focal plane sensors either be affecting the refractive index, the shape, or the position of the optics.

There are five classes of inertial accelerations: static accelerations with respect to their position with the gravity vector, vibrational accelerations from re-pointing, integration and handling, transportation, and seismic loads. For each class of inertial loads, the optics must be able to survive the resultant stresses and deflections, as well as function and perform to specifications. Table 3 shows the loads and requirements placed on the optics.

Table 3. Camera Optics: Structural Load Cases.

\begin{tabular}{|c|c|c|}
\hline & Load Case & Description \\
\hline \multirow{5}{*}{ 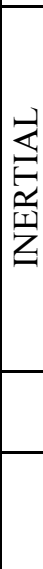 } & $\begin{array}{ll}\text { Gravity (Static) } \\
\mathbf{\square} & \text { Camera Orientation } \\
\mathbf{\square} & \text { Integration and Handling } \\
\text { Transport }\end{array}$ & $\begin{array}{l}\text { With respect to the camera coordinate system, the gravity vector is different for } \\
\text { different camera orientations. The unit gravity vectors in X, Y, and } \mathrm{Z} \text { were } \\
\text { scaled and linear superimposed to envelop all camera orientations and } \\
\text { configurations from integration and test (I\&T), to transport, to installation on the } \\
\text { telescope }\end{array}$ \\
\hline & Repointing (Dynamic) & $\begin{array}{l}\text { Angular velocities and accelerations of the telescope re-pointing creates inertial } \\
\text { loads on the camera, and were combined with the static gravity loads }\end{array}$ \\
\hline & Seismic & $\begin{array}{l}\text { Both SLAC and Chile are seismically active regions. The seismic loads apply to } \\
\text { the camera at all stages of I\&T and in any standard orientations of the camera. }\end{array}$ \\
\hline & Thermal Distortions & $\begin{array}{l}\text { Operating temperatures of the camera can induce stresses and deformations due } \\
\text { to temperature gradients or CTE (Coefficient of Thermal Expansion) mismatches }\end{array}$ \\
\hline & Pressure Differentials & $\begin{array}{l}\text { Pressure loads exist on } \mathrm{L} 3 \text { and } \mathrm{L} 1 . \mathrm{dP} \text { across } \mathrm{L} 3 \text { is from atmospheric }(+101 \mathrm{kPa}) \\
\text { and camera purge pressure (nominally } \sim+0.7 \mathrm{kPa}) \text { outside and vacuum inside the } \\
\text { cryostat. } \mathrm{dP} \text { across } \mathrm{L} 1 \text { is from camera purge pressure. }\end{array}$ \\
\hline
\end{tabular}

Thermal distortions result when materials with different CTEs (coefficients of thermal expansion) and/or thermal gradients are present. For example, fused silica has a very low CTE compared with aluminum (a standard material used for optical filter depositions). When the ambient temperature drops, the two components will contract at different rates. The difference in the interface motion creates stresses in the optics that must be compensated by flexures or other means. Additional details are given in the following sections on design and analysis.

Pressure loading from the atmosphere and/or forced dry, ambient air generates loads on the mechanical interfaces as well as stresses in the optics. The greatest pressure load comes from the atmospheric loading on L3, since the 2nd surface of this optic is in vacuum. As mentioned, there is also a small dry air interior pressure maintained inside the camera body 
in order to prevent dust and other contaminants from accumulating and to keep the optics from developing condensation on their surfaces. This internal pressure exerts pressure loading on the second and first surfaces of L1 and L3.

Finally, internal loads due to manufacturing issues could result in residual stresses. Common causes are machining tolerances, compression of a gasket or O-ring, internal fastener loads, or interference fits. Generally, the loads from the manufacturing sources are low in comparison to other load sources.

In addition to the loads on the optics, the mechanical interfaces are important to evaluate because they define how the optics are mechanically mounted to the various structures. In general, all optical mounts are compliant to distribute the loads and minimize stress concentrations. Additionally, the degrees of freedom are designed to decouple the loads from the rest of the camera. Therefore, external mechanical loads on the camera do not have any primary load paths through any of the optics.

A preliminary, but detailed, structural analysis of the optics has been performed. The stresses and minimum margins of safety are summarized in Table 4.

Table 4. Minimum Margins of Safety for Optical Components and Structures.

\begin{tabular}{|l|l|l|l|l|l|l|}
\hline Component & Load Case & Material & $\begin{array}{l}\sigma \text { allowable } \\
{[\mathrm{MPa}]}\end{array}$ & $\sigma$ calc $[\mathrm{MPa}]$ & $\begin{array}{l}\text { Safety } \\
\text { Factor }\end{array}$ & $\begin{array}{l}\text { Margin of } \\
\text { Safety }\end{array}$ \\
\hline L1 & Camera Pressure & Fused Silica & 52.4 & 2.0 & 7.5 & 2.5 \\
\hline L2 & Gravity & Fused Silica & 52.4 & 0.2 & 7.5 & 34. \\
\hline L1-L2 Assy & Gravity & Aluminum 6061-T6 & 241 & 27.8 & 2.0 & 3.3 \\
\hline Filter & Clamping Load & Fused Silica & 52.4 & 1.9 & 7.5 & 2.7 \\
\hline Filter Frame & Thermal & Titanium 6AL-4V & 827 & 3 & 2.0 & High \\
\hline L3 & $\begin{array}{l}\text { Atmospheric } \\
\text { Pressure }\end{array}$ & Fused Silica & 52.4 & 6.2 & 7.5 & 0.13 \\
\hline $\begin{array}{l}\text { L3 Structural } \\
\text { Ring }\end{array}$ & $\begin{array}{l}\text { Atmospheric } \\
\text { Pressure }\end{array}$ & $\begin{array}{l}\text { Titanium } \\
\text { 6AL-4V }\end{array}$ & 827 & 173. & 2.0 & 1.39 \\
\hline
\end{tabular}

The lenses and filters contribute to the overall optical throughput losses of the camera and, as such, these optics are required to maximize the amount of light that can traverse the fused silica material. Figure 2 shows the expected throughput of the substrate alone for the various optical elements. When applied to the various thicknesses of the different optical elements (lenses and filters), the overall throughput loss due to substrate is very small, (of the order of a few tenths of a percent) and is well within our overall throughput specifications.

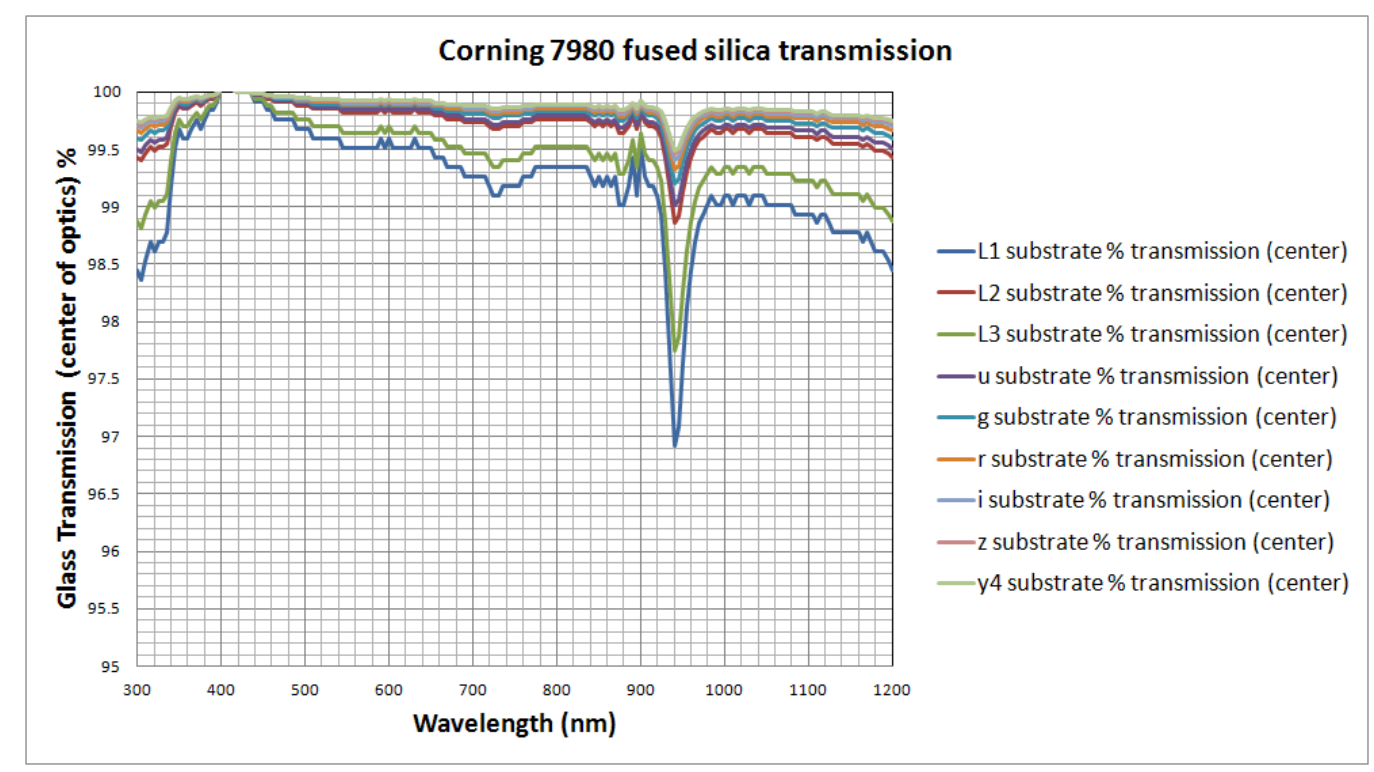

Figure 2. Substrate throughput. 


\subsection{Filter Bandpass and Optical Performance Requirements}

The filter complement will include six filters in the wavelength range limited by atmospheric absorption in the UV and silicon detection efficiency $(320-1050 \mathrm{~nm})$ in the red band, with circular, curved filters with no gaps in the coverage, in order to enable robust and accurate photometric redshifts and stellar typing.

\section{Requirements to be met over integrated incident cone for the central $100 \mathrm{~mm}$ aperture}

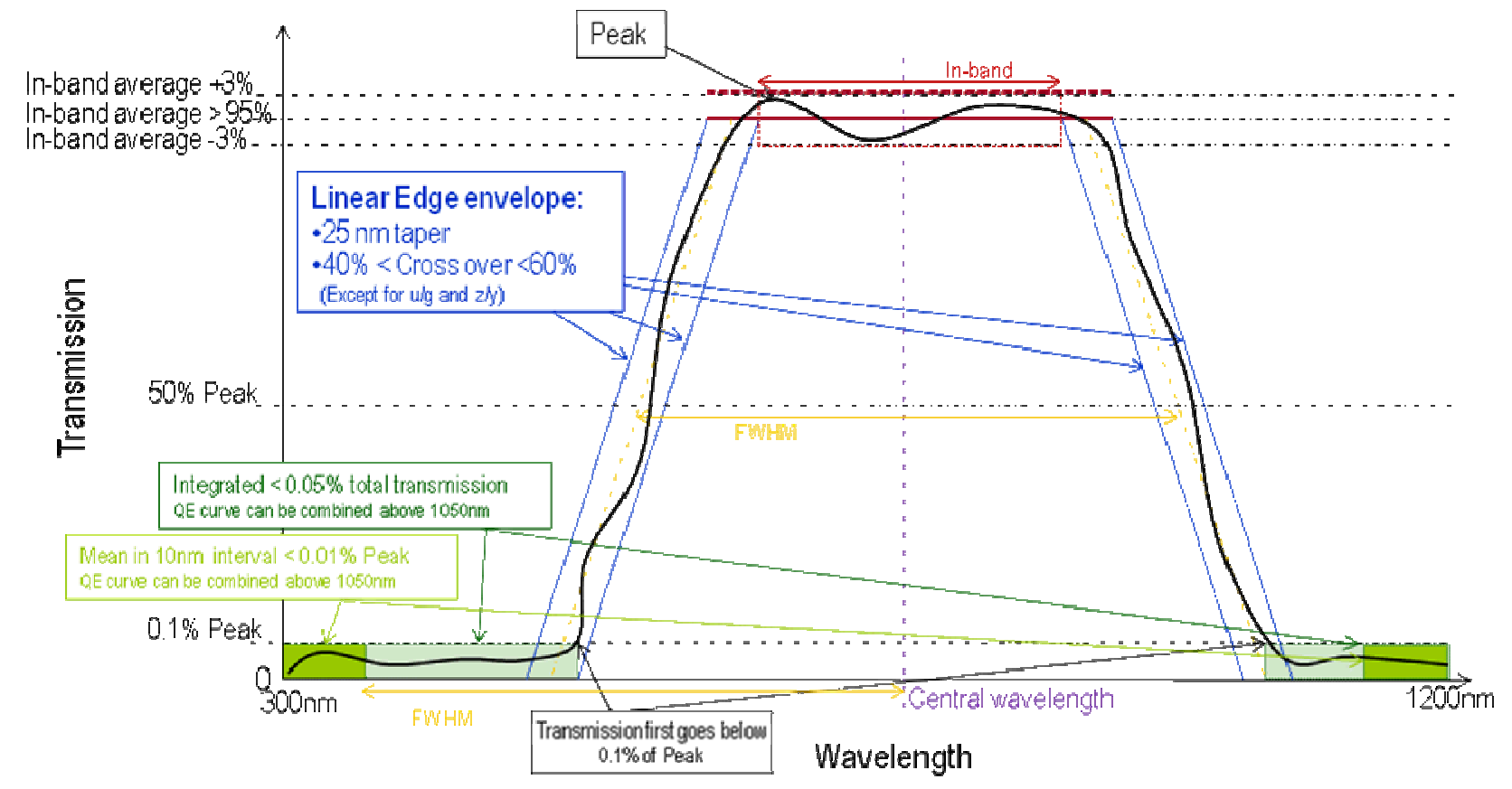

Figure 3. Filter band pass requirements.

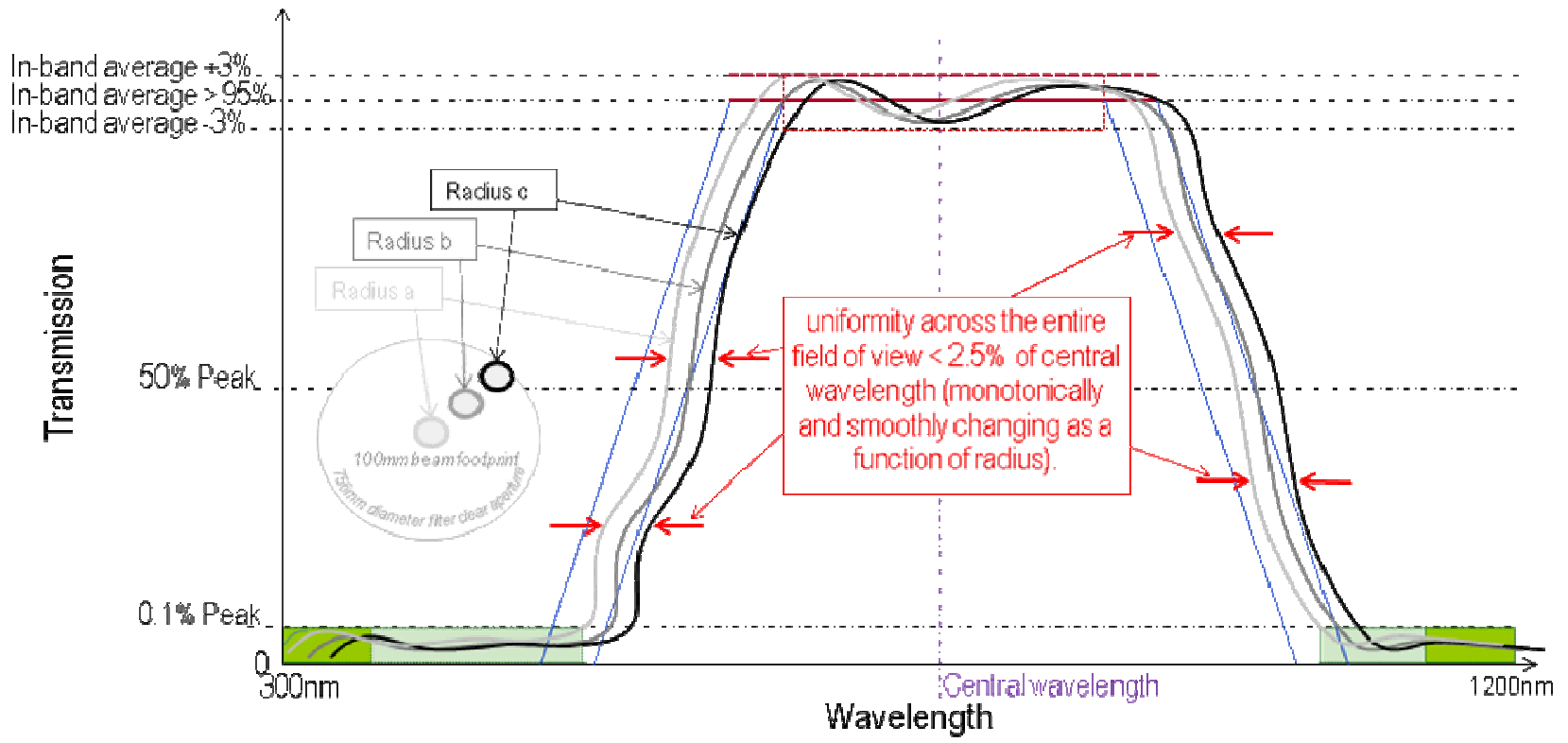

Figure 4. Filter uniformity requirement: Uniformity of the effective wavelength of the filter bandpass (integrated over the incident input cone) for any $100 \mathrm{~mm}$ aperture shall be less than $2.5 \%$ of the nominal central wavelength relative to the nominal wavelength response (integrated over the incident input cone) defined at the central 100mm aperture Nonuniformity assumed to be monotonically changing as a function of radius. 
An SDSS-like $u$ band is extremely important for separating low redshift quasars from hot stars and for estimating the metallicities of $\mathrm{F} / \mathrm{G}$ main sequence stars. A bandpass with an effective central wavelength at about 1 micron will enable studies of substellar objects, high-redshift quasars (to redshifts of 7.5), and regions of the Galaxy that are obscured by interstellar dust. The current LSST filter complement (u, g, r, i, z, y) is modeled on the system used for the SDSS (Fukugita et al. 1996 [5]), which covers the available wavelength range with roughly logarithmic spacing while avoiding the strongest telluric emission features and sampling the Balmer break. Extension of the SDSS system to longer wavelengths (y-band) is possible because the deep depletion CCDs have high sensitivity to 1 micron. The u-band improves the robustness of photometric redshifts of galaxies, stellar population separation, and quasar color selection, and will provide significant additional sensitivity to star formation histories of detected galaxies. The current LSST baseline design has a goal of $1 \%$ relative photometric calibration, which drives the requirements on the filter set. The filter bandpass specifications are based on an envelope definition (see Figure 4) designed to guarantee a good transmission cross-over level, as well as out-of band and in band transmission levels consistent with science performances for photometric redshifts, star separation and star metallicity determination.

Reflection of light by the filter surfaces has to be minimized to maximize throughput through the optical components of the camera. Performance near $300 \mathrm{~nm}$ is the most difficult to achieve due to the dispersion of the materials in the region, hence limiting solutions across the full spectral range. Current interactions with vendors who can provide optics that meet the LSST requirements have shown analysis that the broad band AR coating with a $1 \%$ loss per surface is achievable. Wide band AR coatings typically have, many, very thin layers that are critical to performance. Since these coatings extend into the near UV, those layers are physically very thin (10-200 nm thick) as well as optically thin. During deposition, this translates into a short layer time, which can create an azimuthal non-uniformity (non-uniform points on a constant radius on the optic). This thickness variation, if not taken into account during the deposition processes and chamber geometry, can cause significant spectral non-uniformity and will compromise optical quality. A development phase is planned with potential vendors to arrange the deposition geometry and processes appropriately and we will carefully monitor possible azimuthal variations in the thin layers of the coating in order to address those issues and suggest solutions to the vendors.

\section{L1/L2 ASSEMBLY}

\subsection{L1/L2 Design Description}

The large L1 and L2 lenses are housed in a sub-assembly at the front end of the camera. The L1 lens has a clear aperture radius of $775 \mathrm{~mm}$ and forms the window into the camera volume, with a diameter $(1.57 \mathrm{~m})$ nearly that of the camera itself. With a clear aperture of $551 \mathrm{~mm}, \mathrm{~L} 2$ is significantly smaller in diameter, but still large and heavy. As shown in Figure 5, the two lenses are supported by a toroidal ring with a triangular cross-section; two sides of the ring are open in order to minimize weight. The open-sections are designed to provide maximum stiffness in a compact package, to stay clear of the lens' clear apertures on the inside, while not encroaching on the main light beam from the M2 to M3 mirrors that grazes by on the outside. Each lens is mounted to the ring by flexures that isolate the lenses from forces and moments due to differential expansion of the ring and optics, and also ensures that the glass is supported to provide optical and mechanical stability. The entire subassembly is kinematically supported by a hexapod of adjustable struts that offset the lenses from the front end of the camera body. This assembly provides a stiff support structure for the lenses while minimizing the loads on the glass.

The mechanical design evolves from two driving requirements. First, the physical motions of the lenses must be compatible with the overall camera image quality requirements. When the camera is tilted or rotated, the gravity vector changes relative to the camera coordinate system. All of the optical components will experience differential motion, affecting the image quality. Some correction is possible through camera pointing. A camera-level image quality analysis was performed resulting in mechanical stiffness requirements for the L1/L2 Assembly.. Secondly, the camera total mass and center of gravity requirements drive the assembly structure to be as lightweight as possible. Because this assembly is at the extreme front end of the camera, its mass is offset farthest from the telescope rotator-hexapod assembly. This results in the largest moments and the biggest impact on the top end natural frequency and response to dynamic loads. Given that the size and mass of the lenses cannot be modified, the mass of the support structure must be carefully optimized to reduce unneeded structural mass while still providing adequate stiffness and stability.

The tubular space-frame forming the inner cone of the support ring points to a heritage in aerospace structures. Early frame designs included a sheet metal cone, but structural analysis indicated that the solid cone was not being efficiently 
loaded and perforating the cone with lightening holes would produce curved members not well suited for carrying structural loads. The space-frame provides excellent structural stiffness along with light weight and relatively straightforward manufacturing. The struts are axially loaded and are made from thin-walled aluminum tubes with epoxied end fittings. The entire space-frame is considerably lighter than the original solid cone, while still providing adequate stiffness to react the combined shear and torsional loads.
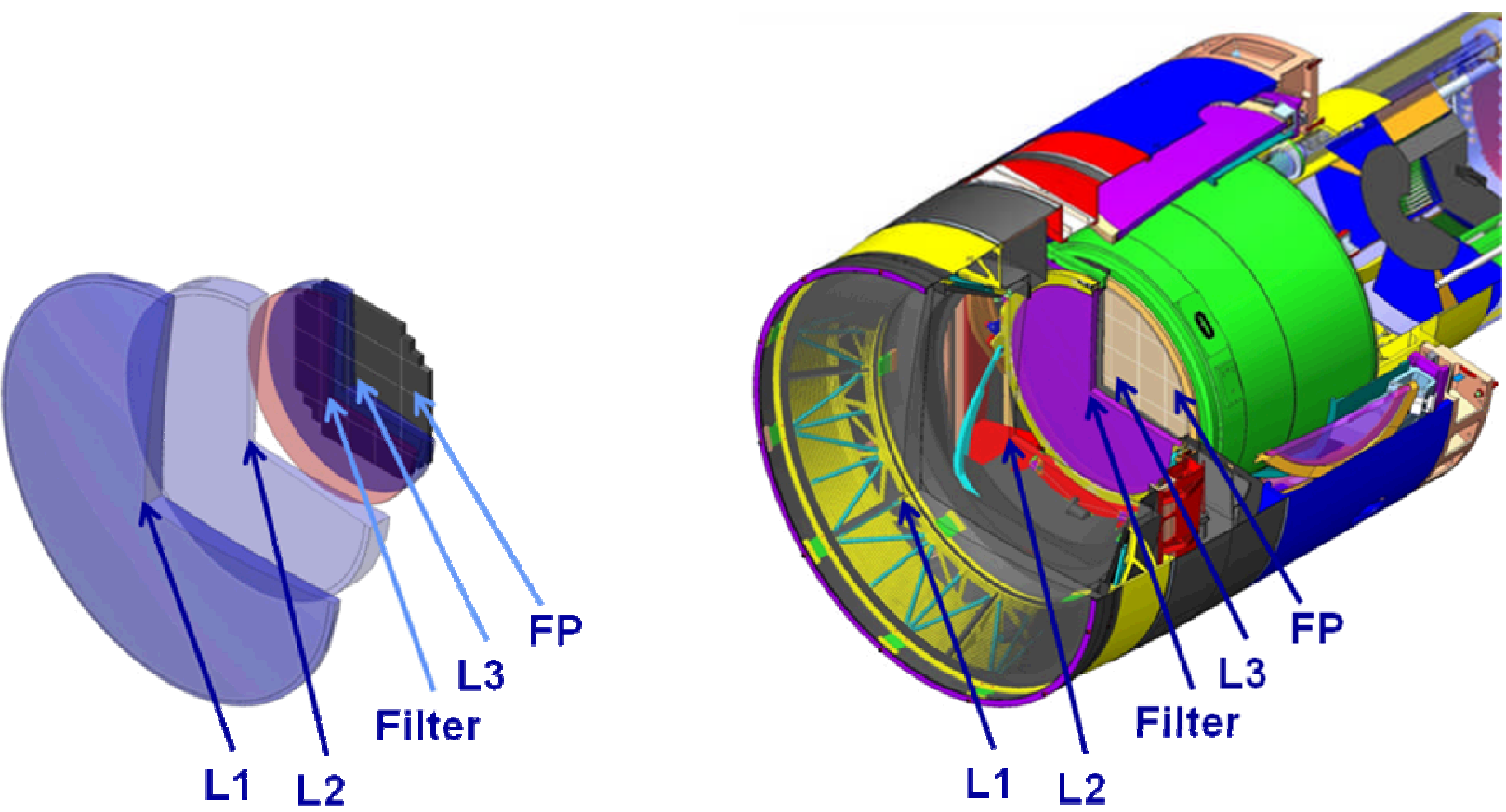

Figure 5. Optical Components shown in camera 3D model.

The lens assembly mounting struts form the final element of the structural design. These support the mass of the entire assembly off of the front of the camera housing. Their mounting locations have been optimized to reduce the impact of the point-loading on the deflection of the lenses. In particular, the struts are actually mounted in the interior of the toroidal ring, near the neutral axis of the triangular cross-section. The shape of the cross-section has been adjusted so that the shear center and center of gravity were brought in line with the neutral axis. This ensures that the cone is supported in a location to minimize deflections from both out-of-plane bending as well as warping from in-plane shear..

\subsection{Mechanical Design \& Analysis}

The L1/L2 lenses are large glass elements at the far end of the camera. In order to minimize moments at the camera to telescope interface, the structure was designed to be as lightweight as possible. When subjected to the load cases discussed in the previous section, the glass exerts loads into the radial flexures, which act on the structural ring. The structural ring carries the shear and torsional loads to three heavy duty brackets, which are connected to the six struts that form the kinematic L1/L2 Assembly mount. The six strut axial forces are then reacted into the camera body's front flange. Depending on the camera elevation and rotation angle, the loads in each structural element varies. In order to envelope every possible orientation, a $1 \mathrm{G}$ gravity was applied to the camera X, Y, and Z-axes. Using linear superposition, an envelope of every possible orientation was calculated. The resulting interface loads and components stresses were calculated along with margins of safety.

Because the camera is hermetically sealed from its surroundings and has a small positive internal pressure, there is a pressure gradient pushing outward on the L1 lens. L2 is fully contained inside the camera, so it does not experience any pressure gradients. The resulting stresses from the internal pressure load were calculated and superimposed with the intertial and thermal load results. 


\subsection{Optics Interfaces}

The most significant interface occurs between the lenses and the toroidal structural ring. Because these two materials have very different CTE's, it is important to use radially oriented flexures to minimize thermally induces stresses. The problem occurs when there is a decentering load on the lenses, it creates a stress field that is not axisymmetric, but the stress levels are low enough that image quality is not severely impacted.

Another interface that is very important is the orientation of the six struts. These struts are designed to act like three Aframes, creating a kinematic mount for the L1/L2 ring. A difficulty arises because the struts cannot mechanically meet at a sharp point. To address this physical limitation, the virtual intersection point of the strut lines-of-action are located at the cross section shear center. This minimizes the amount of twisting in the toroidal ring.

\subsection{Component Manufacturing}

L1 is composed of two spherical surfaces (one convex and one concave). The front side of L2 is plano while the back side is a 6th order polynomial asphere. The aspheric departure of L2 is just below 300 um relative to a sphere fit to the vertex and edge of the lens and was traded off with the secondary mirror aspheric departure for ease of fabrication. Inspection polish on L1 and L3 edges and bevels are prudent in order to avoid providing a failure starting point for these under-pressure components. The wedge is typically measured by total indicator movement. The lenses and filters have alignment specifications of $\sim 100$ microns, which are easily measured in standard setups. The use of power spectral density for specifying wavefront is a way to control the ripple or "waviness" that tends to result when an optic is fabricated with small-tool polishing, as is often the case for aspheres. Peak-to-valley and rms specifications by themselves do not sufficiently control the wavefront quality and could result in unacceptable image quality and/or distortion artifacts. As an example, single-point diamond turning can result in a distinct rippled "signature" which can cause undesirable artifacts in the image.

\subsection{Assembly and test}

Spherical surfaces are the easiest to test since an interferometer fitted with a transmission sphere can produce an accurate spherical wavefront normally-incident to the optic-under-test. Even if the transmission sphere does not produce a perfect spherical reference, any reasonable deviation can be calibrated out using well-established absolute sphere testing techniques.

Testing aspheric surfaces is more difficult. Traditionally, separate null lenses were designed and built to test aspheric surfaces. The null lenses were such that the combination of the null lens and the lens under test $t$ would produce a spherical wavefront. These null lenses were usually fabricated using spherical surfaces which were relatively easy to test and assemble. The additional steps involved, however, create increased opportunity for error. The primary mirror of the Hubble Space Telescope is the most vivid example of the mischief that can be caused by the additional complexity of a null lens.

Other techniques reduce the likelihood of error. For small amounts of departure from spherical shapes and slopes, the known departure can be subtracted out via a calculated reference file. For larger aspheric departures or very high precision smaller aspheric departures, one needs to take into account ray re-trace errors: if a spherical wavefront is incident onto an aspheric surface, then the reflected wavefront will not follow exactly the same path on the return trip, a common approximation in interferometry. This can be managed with analysis. Large aspheric departures can be handled with other techniques such as sub-Nyquist interferometry.

In LSST, the approach taken was to design the optical component null tests simultaneously with the design of the telescope. As a result, each optic has a test that uses an incident spherical or flat wavefront and produces a spherical or flat wavefront as though spherical surfaces were being tested. Vendors could choose a different testing technique that is more suited to their capabilities, but the designed null test provides confidence that the optic can be tested.

A null test also exists for the L1/L2 assembly. The null test includes a plane parallel window which is $105.2 \mathrm{~mm}$ thick. The position of the window is not crucial. The purpose of the window is to add the correct amount of spherical aberration to null the expected wavefront. Alternatively, the test could be performed without the window with a $\sim 15.4$ wave $\mathrm{P}-\mathrm{V}$ spherical aberration offset in the interferometer reference file. 


\section{4. $\mathbf{L 3}$}

\subsection{L3 Design Description}

The L3 lens assembly is the vacuum barrier for the cryostat containing the detector array. The assembly is mechanically fastened to the front flange of the cryostat housing with a ring of screws. A double O-ring seal ensures a tight vacuum seal. Figure 6 shows a cross sectional view of the L3 Assembly and its interface to the Cryostat housing.

The three structural components to this assembly are the L3 Lens, the structural ring, and the retaining ring. The L3 lens is the window of the cryostat and carries the atmospheric pressure loads. These pressure loads find a load path into the structural ring, the second structural component. This structural ring is a thick annular ring machined from Titanium alloy, capable of carrying the high annular bending stresses. The final structural component is the retaining ring, which serves to stabilize the L3 lens in its mount.

\subsection{Mechanical Design \& Analysis}

The L3 assembly does experience inertial accelerations and thermally induced loads, but the largest stresses, by far, come from the atmospheric pressure loads. The forces due to inertial accelerations are a function of the L3 mass. This results in relatively low maximum forces $(0.8 \mathrm{kN}$ max $)$. The thermal distortion between the metallic structural ring and the lens is significant, but because the actual mount is Viton, a compliant material, the stresses generated by thermal distortion are also low (resulting stress $<1 \mathrm{MPa}$ ).

The atmospheric loads can be enveloped by assuming a complete vacuum on the interior volume of the cryostat $(\mathrm{Pi}=0)$ and the worst case atmospheric pressure on the outside ( $\mathrm{Po}=$ lowest elevation where vacuum is pulled). The atmospheric pressure at SLAC is $101 \mathrm{kPa}$ and envelopes the worst case external pressure the L3 assembly will experience. In addition to the atmospheric pressure, there is also a small over pressure from the purge system. Accounting for worst case overpressure, the maximum pressure is $102 \mathrm{kPa}$. Over the area of L3, the total force is calculated to be $43 \mathrm{kN}$.

Implosion is a serious concern because it could result in damage to the science sensors or the glass could fall out of its mount onto the shutter or other camera components. Some conservative fracture analyses have been performed to ensure that 1) there is adequate margin of safety against fracture, and 2) if there is a fracture, L3 will not implode, but instead jam in its mount and allow safe re-pressurization of the cryostat.

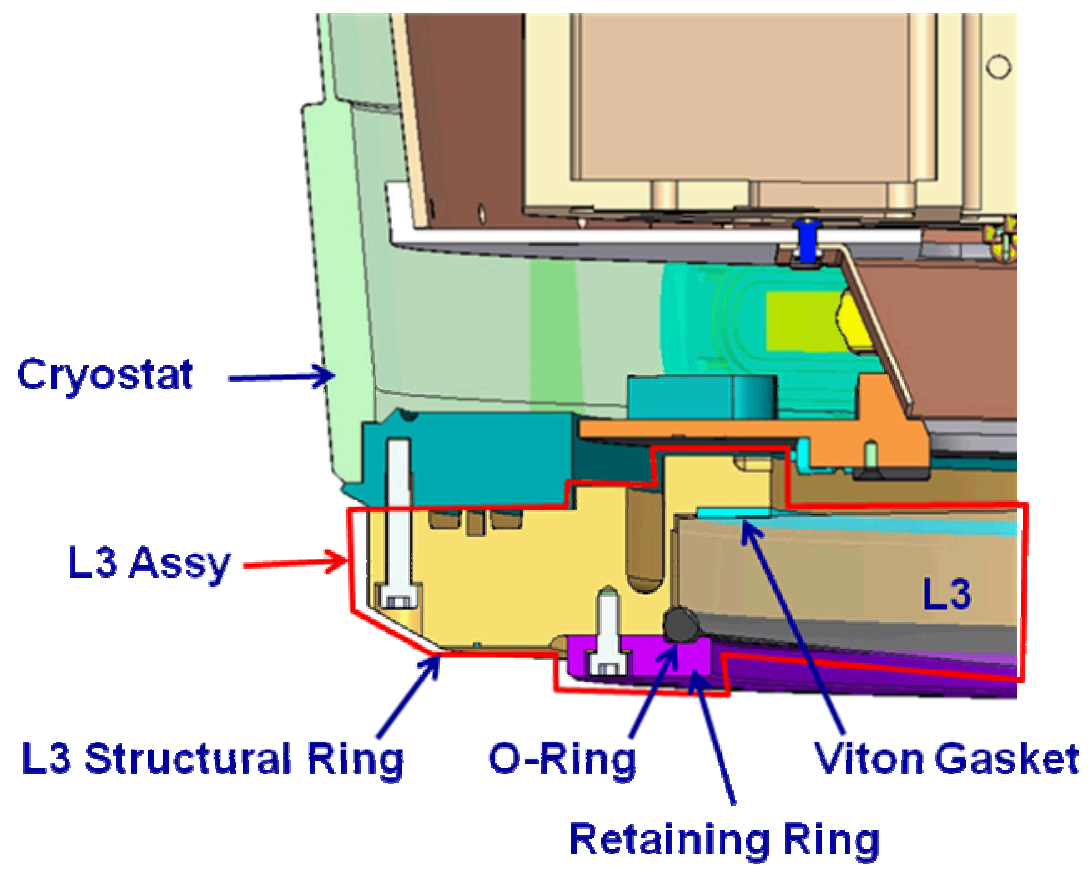

Figure 6. L3 Assembly mechanical components. 
The pressure loads find a load path into the structural ring through a Viton gasket. A detailed FEA model of this interface was studied and it was found that the compressive stresses in the Viton gasket are within the material constraints; the structural ring, a thick Titanium annulus, is also capable of carrying the high annular bending stresses.

The final structural component is the retaining ring, which primarily serves to stabilize the L3 lens in its mount. These stresses are very low and have correspondingly high margins of safety. The stress analysis of L3 and minimum margins of safety are summarized in Table 5.

Table 5. L3 Minimum Margins of safety.

\begin{tabular}{|c|c|c|c|c|c|c|}
\hline Load Case & Component & Material & $\begin{array}{l}\sigma \text { allowable } \\
\text { [MPa] }\end{array}$ & $\begin{array}{l}\sigma \text { calc } \\
{[\mathrm{MPa}]}\end{array}$ & $\begin{array}{l}\text { Safety } \\
\text { Factor }\end{array}$ & $\begin{array}{l}\text { Margin of } \\
\text { Safety }\end{array}$ \\
\hline Atmospheric Pressure & L3 & Fused Silica & 52.4 & 6.2 & 7.5 & 0.13 \\
\hline Atmospheric Pressure & $\begin{array}{l}\text { L3 Structural } \\
\text { Ring }\end{array}$ & Titanium 6AL-4V & 827 & 173. & 2.0 & 1.39 \\
\hline Thermal Load & L3 & Fused Silica & 52.4 & 0.7 & 7.5 & 8.98 \\
\hline $\begin{array}{l}\text { Combined Atm + } \\
\text { Thermal }\end{array}$ & L3 & Fused Silica & 52.4 & 5.5 & 7.5 & 0.27 \\
\hline
\end{tabular}

4.3 Optics Interfaces

As discussed briefly above in section 4.2, the L3 lens does not contact metal directly - the mounting is via two elastomeric parts. To transfer the atmospheric pressure loads, there is a flat gasket between the lens and the Titanium structural ring. The width and thickness of the gasket was designed to minimize stress concentrations. The second interface is an O-ring between a chamfer in L3 and the titanium retaining ring. This O-ring not only provides centering and stabilization of L3, but also forms a vacuum seal for the annular volume at the lens edge. This interface is detailed in Figure 6. The gasket and O-ring materials are both Viton, chosen for its compatibility with vacuum, excellent compression-set characteristics, temperature operating range, and heritage in previous vacuum vessel applications.

\subsection{Component Manufacturing}

As with the other elements, the substrate is fused silica with a convex conic on the front side and a convex sphere on the other side. L3 is designed such that a correctly-positioned point source on incident on the back side will produce a perfectly-corrected beam. The aspheric departure is modest (small) for such a large optic: at most about 14 microns when fabricated using a best-fit sphere. The aspheric departure is small enough so that this surface can be tested with a spherical beam if there is a sufficiently large transmission sphere. The null test for L3 is particularly attractive; however, the only large optic required is a flat mirror. Absolute flat testing techniques reduce the requirements for the wavefront quality of the flat mirror.

\subsection{Assembly and test}

Two interferometric tests are required to guarantee compliance. One surface should be tested in reflection-always a spherical or flat surface. Then the null test should be performed that takes into both surfaces. The aspheric departure on L3/S1 is small enough so that the surface can be tested by itself in reflection by using a "dry-labbed" reference file. The maximum departure of 14 microns is only about 20 fringes, well within the ability of a modern interferometer to analyze.

\section{FILTERS}

\subsection{Filter Mechanical Design \& Analysis}

The filters are mounted in a titanium frame that holds the glass steady while minimizing stress, as well as providing the mechanical interfaces to move the filters, and the fiducials required to locate the filters in the online position within the required precision tolerances. The frame comprises two precision machined titanium rings. The first ring is structural and is the same for all filters. It serves four primary purposes: 1) locates the filter with respect to the first surface; 2) contains the mechanical fiducials used in the repeatable interface; 3 ) provides interface to Auto Changer, Carousel and Loader mechanisms; and 4) provides the structural frame to minimize loads into the filter. The second ring is a retaining ring that provides the required clamping force to immobilize the filters in the frame. There is an elastomeric gasket and o-ring to mount the filter to the frame. Figure 7 shows a cross-sectional view to detail the filter mount. 

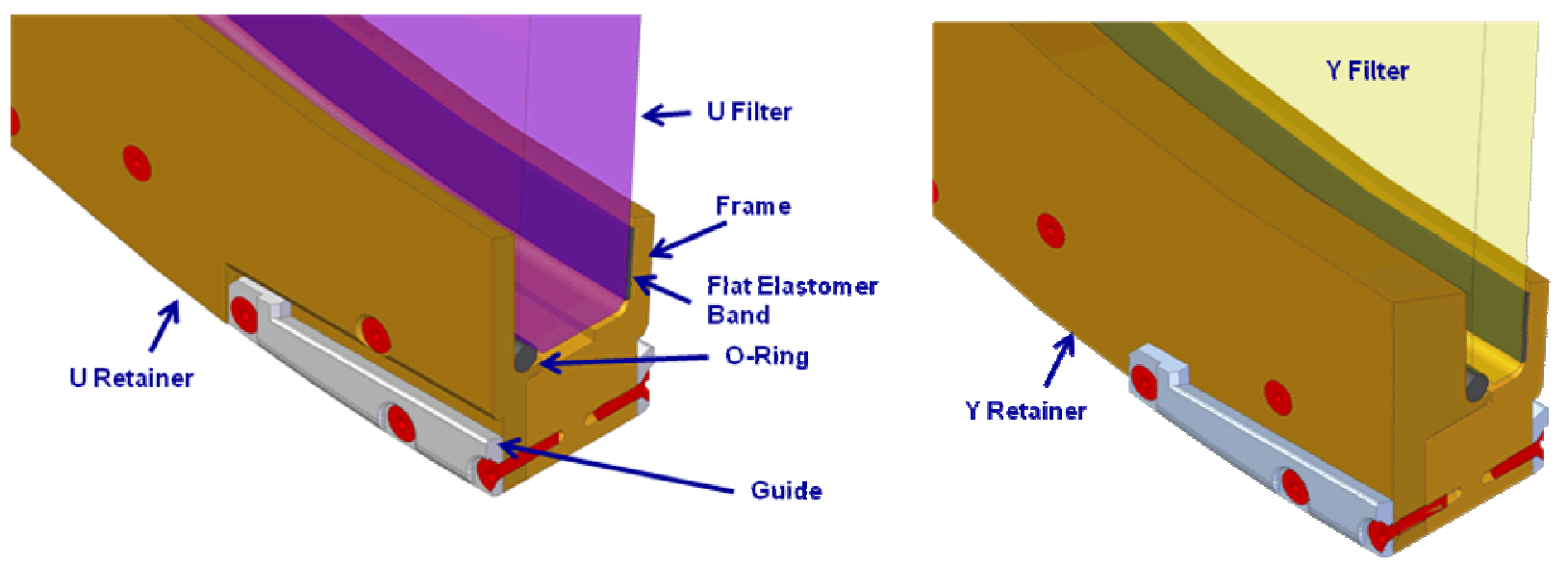

Figure 7. Filter Interface details.

When the retaining ring is fastened to the structural ring, the gasket and O-rings are compressed, imparting stress into the filter. Thermal distortion will also result in stresses in the filters. The main concern in these stresses is that they could either damage the filter coatings or result in a stress-induced index change in the glass. Both of these effects were examined carefully via FEA and found to be of no concern. Another potential concern is that the online position is somewhat over-constrained because there are four restraints in the out-of-plane direction. If these interface points should distort as a result of a changing gravity vector, loads would be imparted into the filters. These worst case loads were accounted for through the full camera FEA, and the resulting loads were found to be of no concern. The standard set of inertial loads experienced during operation was also examined.

\subsection{Filter Coating Design}

The filter coating is a relatively sophisticated multi-layer interference coating that is designed to transmit only light with wavelengths in a specified band, and to reject light at other wavelengths with a specified fidelity. The main challenge is to deposit uniform coatings with the desired characteristics on the large, curved substrates typically with about 100 layers on each surface.

The initial design of the LSST filters was performed with TFCALC to produce bandpass curves that meet the LSST scientific objectives. Multilayer thin film coatings are applied to filters to control the reflected, transmitted, and absorbed light at wavelengths ranging from x-ray to far infrared. A coating's effect is determined by how its layers control the interference and absorption of light.

The current LSST baseline design has a goal of $1 \%$ relative photometry and, as such, defines the general features of the filter set with the following design considerations:

1. Beam that is incident on the detector has a focal ratio of $\mathrm{f} / 1.234$ with a $61.5 \%$ obscuration.

2. The filter is concentric about the chief ray so that all portions of the filter see the same angle of incidence range, about $14.2^{\circ}$ to $23.6^{\circ}$.

3. No gaps should exist between filter band-passes.

4. Filter band-passes should not overlap.

5. Band-pass throughput should be as high possible.

6. The transition between stop and pass band should be less than $5 \%$ of the filter band-pass.

\subsection{Filter development plan}

The LSST filters are significantly large compared to previous systems and have a clear aperture of $756 \mathrm{~cm}$. Their coating uniformity and band-pass performances have been identified at the project level to be a high risk item because of the 
uncertainty in meeting all of the requirements. Because of this risk, the project has established a comprehensive development plan with a primary goal set toward reducing risks related to the fabrication of the filters. This plan is broken down in the following phases:

1. Design Study phase with leading vendors in the industry: The LSST camera project has conducted design studies with 3 vendors leading the industry across the world. One vendor JDSU Uniphase is located in the USA, the second vendor SAGEM is located in France and the third vendor Asahi Spectra is located in Japan. The Laboratoire des Matériaux Avancés (LMA) was in charge to oversee the filters design study for SAGEM. The result of this design study provided a better understanding of the critical issues in the filters coating requirements and manufacturability. The results obtained demonstrated feasibility of the coating uniformity based on previous experience as well as overall feasibility of the bandpass performances. However, the shape of the bandpass as well as out-of-band rejection has been identified as still a challenging task of the filter fabrication for all vendors. Additionally, process variability on the coating transmission features was identified as a critical issue. Given the high cost of the filter (blank and polishing) before coating, it is imperative to ensure that the coating meets all requirements in a single attempt.

2. Coating process development to achieve $1 \%$ variability required for bandpass shape performances. This phase involves working with vendors towards an equipment upgrade and demonstration of the required variability. The equipment upgrade includes improvements of the control of deposition geometries and advanced on-part monitoring for precise placement of edges at multiple wavelengths and multiple positions on the filter.

3. Prototype filter. During this phase, selected vendors will produce small witness samples coated at simulated location on the filter. This phase will demonstrate performances of two representative filters: u-band filter due to its critical repeatability and bandpass shape accuracy; r-band filter as a representative filter demonstration edge control on two sides. The LMA has also conducted some positive tests on small 1 inch substrates for the $r$ band filter.

4. First article of one filter. During this phase, selected vendors will produce a full size first article filter. This phase intends to demonstrate the full process from blank procurement, polishing and coating to ensure that the process expected to occur at different vendors is fully understood. Additionally, the large size coating performances in terms of shape and uniformity will be demonstrated early.

Vendors have showed interest into conducting this development plan and indicated that it could be conducted in 12 to 15 months, which aligns well with the overall schedule of the LSST Camera project.

\subsection{Component Manufacturing, Assembly and test}

Each filter is a thin meniscus with similar (and in some cases, identical) radii of curvature on the two sides. The filters share a common radius of curvature on their convex sides. Thicknesses vary in order mostly to correct the spherical aberration for the wavelength band of that filter. The chief rays are perpendicular to the front surface, and nearly so on the back surface $\left(<0.1^{\circ}\right)$ so that there is minimal field dependence of the filter coating performance. The optics are thin but manufacturable, as indicated in vendor quotes. Since the filter has almost zero power and is nearly concentric about the pupil, there is little effect from bending due to changing gravity vector. The filter null tests have the same basic setup, with minor distance changes from filter to filter

\section{SUMMARY}

We have developed a baseline design of the LSST camera optics and have discussed optics fabrication issues with vendors and found that a substantial industrial base exists for the optics fabrication. We have also discussed the optics coating issues with vendors and found that an adequate industrial base exists for optics coating. We have developed a project execution plan, including budget and schedule for completion of the LSST camera optics. Filter vendors have provided design studies for the six LSST filters which are currently being evaluated. Extensive design and analysis has been performed on the mechanical interfaces to the optical and filter elements. Stress analysis (FEA) has been performed. Work continues to complete the RFQ for the filter coating risk reduction study. 


\section{ACKNOWLEDGEMENT}

LSST is a public-private partnership. Funding for design and development activity comes from the National Science Foundation, private donations, grants to universities, and in-kind support at Department of Energy laboratories and other LSSTC Institutional Members. This work is supported by in part the National Science Foundation under Scientific Program Order No. 9 (AST-0551161) and Scientific Program Order No. 1 (AST-0244680) through Cooperative Agreement AST-0132798. Portions of this work are supported by the Department of Energy under contract DE-AC0276SF00515 with the Stanford Linear Accelerator Center and contract DE-AC52-07NA27344 with Lawrence Livermore

\section{NATIONAL LABORATORY. REFERENCES}

[1] M. Paul, Revue d'optique théorique instrumentale, Vol. 14, No. 5, p. 169 (1935)

[2] J.G. Baker and George Z. Dimitroff, "Telescopes and Accessories", 1945, Blakiston

[3] Rumsey, N.J. 1969, "A Compact Three-Reflections Astronomical Camera, in Optical Instruments and Techniques", ICO8 Meeting, London, Home Dickson Edt. (Oriel Press Newcastle), 514

[4] C.G. Wynne, "Field correctors for astronomical telescopes", Progress in Optics, Vol. X, edited by E. Wolf (1972)

[5] Fukugita, M., Ichikawa, T., Gunn, J.E., Doi, M., Shimasaku, K., and Schneider, "The Sloan Digital Sky Survey Photometric System", D.P. 1996. AJ, 111(4), 1748-1756. 